\title{
Treadmill exercise alleviates nigrostriatal dopaminergic loss of neurons and fibers in rotenone-induced Parkinson rats
}

\author{
Mal-Soon Shin', Tae-Woon Kim², Jae-Min Lee ${ }^{2}$, Eun-Sang Ji², Baek-Vin Lim ${ }^{3, *}$ \\ ${ }^{1}$ School of Global Sport Studies, Korea University, Sejong, Korea \\ 2Department of Physiology, College of Medicine, Kyung Hee University, Seoul, Korea \\ ${ }^{3}$ Division of Leisure \& Sports Science, Department of Exercise Prescription, Dongseo University, Busan, Korea
}

Parkinson disease is one of the common brain diseases caused by dopaminergic neuronal loss in the substantia nigra and dopaminergic fiber loss in the striatum. In the present study, the effects of treadmill exercise on motor performance, dopaminergic loss of neurons and fibers, and $\alpha$-synuclein expression in the nigrostriatum were evaluated using rotenone-induced Parkinson rats. For the induction of Parkinson rats, $3-\mathrm{mg} / \mathrm{kg}$ rotenone was injected, once a day for 14 consecutive days. Treadmill running was conducted for 30 min once a day during 14 consecutive days. Rota-rod test for motor balance and coordination and immunohistochemistry for tyrosine hydroxylase and $\alpha$-synuclein in the nigrostriatum were performed. In the present study, motor balance and coordination was disturbed by induction of rotenone-induced Parkinson disease, in contrast, treadmill exercise alleviated motor dysfunction in the rotenone-induced Parkinson rats. Nigrostriatal dopaminergic loss of neurons and fibers was occurred by induction of rotenone-induced Parkinson disease, in contrast, treadmill exercise alleviated nigrostriatal dopaminergic loss of neurons and fibers in the rotenone-induced Parkinson rats. $\alpha$-Synuclein expression in the nigrostriatum was enhanced by induction of rotenone-induced Parkinson disease, in contrast, treadmill exercise suppressed $\alpha$-synuclein expression in the rotenone-induced Parkinson rats. Treadmill exercise improved motor function through preservation of nigrostriatal dopaminergic neurons and fibers and suppression of nigrostriatal formation of Lewy bodies in rotenone-induced Parkinson rats.

Keywords: Parkinson disease, Rotenone, Treadmill exercise, Substantia nigra, Striatum

\section{INTRODUCTION}

Parkinson disease is one of the common brain diseases caused by dopaminergic neuronal loss in the pars compacta of substantia nigra and dopaminergic fiber loss in the striatum (Burkhardt and Weber, 1994; Ehringer and Hornykiewicz, 1998). Its characteristic symptoms are resting tremor, muscle rigidity, and bradykinesia. Parkinson disease also induces autonomic dysfunction and depression (Berrios et al., 1995).

Rotenone is a retinoid chemical extracted from root of Lonchocarpus species, and it has been used as a pesticide. Rotenone freely enters into the cells (Talpade et al., 2000), binds to complex I in the mitochondria, and then it inhibits electron transfer system (Schuler and Casida, 2001). Chronic exposure to rotenone causes symptoms of Parkinson disease and also aggregates synuclein, forming the Lewy body-like cytoplasmic inclusions (Betarbet et al., 2000).

Tyrosine hydroxylase (TH) is a rate-limiting enzyme in the synthesis of the catecholamine neurotransmitters. TH activity is progressively decreased following the loss of dopaminergic neurons in the substantia nigra of the Parkinson disease (Cho et al., 2013; Yoon et al., 2007). a-Synuclein is a synuclein protein of unknown function primarily found in neural tissue, making up as much as $1 \%$ of all proteins in the cytosol of brain cells (Iwai et al., 1995).
${ }^{\star}$ Corresponding author: Baek-Vin Lim (iD http://orcid.org/0000-0003-4017-573X Division of Leisure \& Sports Science, Department of Exercise Prescription, Dongseo University, 47 Jurye-ro, Sasang-gu, Busan 47011, Korea Tel: +82-51-320-1887, Fax: +82-51-320-1945, E-mail: todd64@naver.com Received: January 2, 2017 / Accepted: February 1, 2017
This is an Open Access article distributed under the terms of the Creative Commons Attribution Non-Commercial License (http://creativecommons.org/licenses/by-nc/4.0/) which permits unrestricted non-commercial use, distribution, and reproduction in any medium, provided the original work is properly cited. 
a-Synuclein is predominantly expressed in the neocortex, hippocampus, substantia nigra, thalamus, and cerebellum. Oxidation of $a$-synuclein composes one of the components of Lewy body (Giasson et al., 2000), and then a-synuclein in solution is considered to be an intrinsically disordered protein, i.e., it lacks a single stable 3-dimensional structure (van Rooijen et al., 2009).

Exercise is known to improve motor performance, enhance memory function, and reduce progression of Parkinson disease (Chen et al., 2005; Miyai et al., 2000). Motor training attenuated behavioral and neurochemical deficits in 6-OHDA-induced Parkinson rats (Cohen et al., 2003; Tillerson et al., 2003). Special movement therapy improved short-term memory in Parkinson patients (Schalow et al., 2005).

In the present study, we investigated whether exercise exerts therapeutic effect on Parkinson disease. For this, the effects of treadmill exercise on motor performance, dopaminergic neuronal loss and $\alpha$-synuclein expression in the nigrostriatum were evaluated using rotenone-induced Parkinson rats.

\section{MATERIALS AND METHODS}

\section{Animals and treatments}

Adult male Sprague-Dawley rats weighing 200 10 g (6 weeks in age) were used in this experiment. The rats were housed at a room temperature $\left(20^{\circ} \mathrm{C} \pm 2^{\circ} \mathrm{C}\right)$ under standard $12 \mathrm{hr}$ light/dark cycles (lights on at 07:00 a.m.). Food and water were made available ad libitum. The experimental procedures were performed in accordance with the animal care guidelines of the National Institutes of Health and the Korean Academy of Medical Sciences. The animals were randomly assigned into four groups ( $\mathrm{n}=10$ in each group): the control group, the control exercise group, the rotenone-injection group, and the rotenone-injection and exercise group. Rotenone was dissolved in a mixture of dimethyl sulfoxide:polyethylene glycol (1:1). Rotenone at $3 \mathrm{mg} / \mathrm{kg}$ was injected to rats in the rotenone-injection groups, once a day for 14 consecutive days.

\section{Exercise protocol}

The animals in the exercise groups were forced to run on a motorized treadmill for $30 \mathrm{~min}$ once a day during 14 consecutive days. The exercise load consisted of running at a speed of $2 \mathrm{~m} / \mathrm{min}$ for the first $5 \mathrm{~min}, 3 \mathrm{~m} / \mathrm{min}$ for the next $5 \mathrm{~min}$, and then $4 \mathrm{~m} /$ $\min$ for the last $20 \mathrm{~min}$.

\section{Rota-rod test}

Rota-rod (Harvard Apparatus, Holliston, MA, USA) test for motor balance and coordination was conducted, according to the previous study (Cho et al., 2016). Each rat was placed in a separate compartment on the rotating rod (diameter, $7 \mathrm{~cm}$ ). The velocity of the rod was set at a constant $5 \mathrm{rpm}$. Latency until fall was automatically recorded by magnetic trip plates. Maximum cutoff latency was set as $180 \mathrm{sec}$.

\section{Tissue preparation}

After rota-rod test, the animals were deeply anesthetized with Zoletil $50(10 \mathrm{mg} / \mathrm{kg}$, intraperitoneally; Vibac Laboratories, Carros, France), transcardially perfused with $50-\mathrm{mM}$ phosphate-buffered saline (PBS) and fixed with $4 \%$ paraformaldehyde in 100$\mathrm{mM}$ phosphate buffer at $\mathrm{pH}$ 7.4. The brain were removed, postfixed in the fixative overnight, and transferred into a 30\% sucrose solution for cryoprotection. Serial coronal sections of $40 \mu \mathrm{m}$ thick were made with a freezing microtome (Leica, Nussloch, Germany).

\section{Immunohistochemistry for TH in the nigrostriatum}

The sections in the substantia nigra was selected from the region spanning from Bregma -5.2 to -5.6 mm, and TH immunohistochemistry was performed, as the previously described method (Cho et al., 2013; Yoon et al., 2007). The staining was carried out using free-floating sections. The sections were rinsed in PBS and incubated in $1 \% \mathrm{H}_{2} \mathrm{O}_{2}$ for 30 min to block the endogenous peroxidase activity. After washing in PBS, the sections were incubated in blocking serum (10\% normal horse serum and $0.1 \%$ Triton $\mathrm{X}-100$ in PBS) for $60 \mathrm{~min}$, followed by incubation in anti-TH mouse monoclonal antibody solution (1:500, Oncogene Research Product, Cambridge, UK) for $24 \mathrm{hr}$ at room temperature. The sections were then incubated for $1 \mathrm{hr}$ in biotinylated anti-mouse IgG secondary antibody (1:300; Vector Laboratories, Burlingame, CA, USA). The sections were subsequently incubated with avidin-biotin-peroxidase complex (Vector Laboratories) for $1 \mathrm{hr}$ at room temperature. Immunoreactivity was visualized by incubating the sections in a solution consisting of $0.05 \%$ 3,3-diaminobenzidine (DAB) and $0.02 \% \mathrm{H}_{2} \mathrm{O}_{2}$ in $50-\mathrm{mM}$ Tris buffer $(\mathrm{pH}$, 7.6) for $3 \mathrm{~min}$. The sections were mounted on gelatine-coated slides and cover slipped with mounting medium.

For TH-immunohistochemistry in the striatum, the sections from Bregma 0.1 to $-0.1 \mathrm{~mm}$ were selected to quantify the optical densities of $\mathrm{TH}$-immunoreactive fibers, according to the previously described method (Cho et al., 2013; Yoon et al., 2007). TH-immunoreactive fiber density was measured in $100 \mu \mathrm{m} \times 100$ 

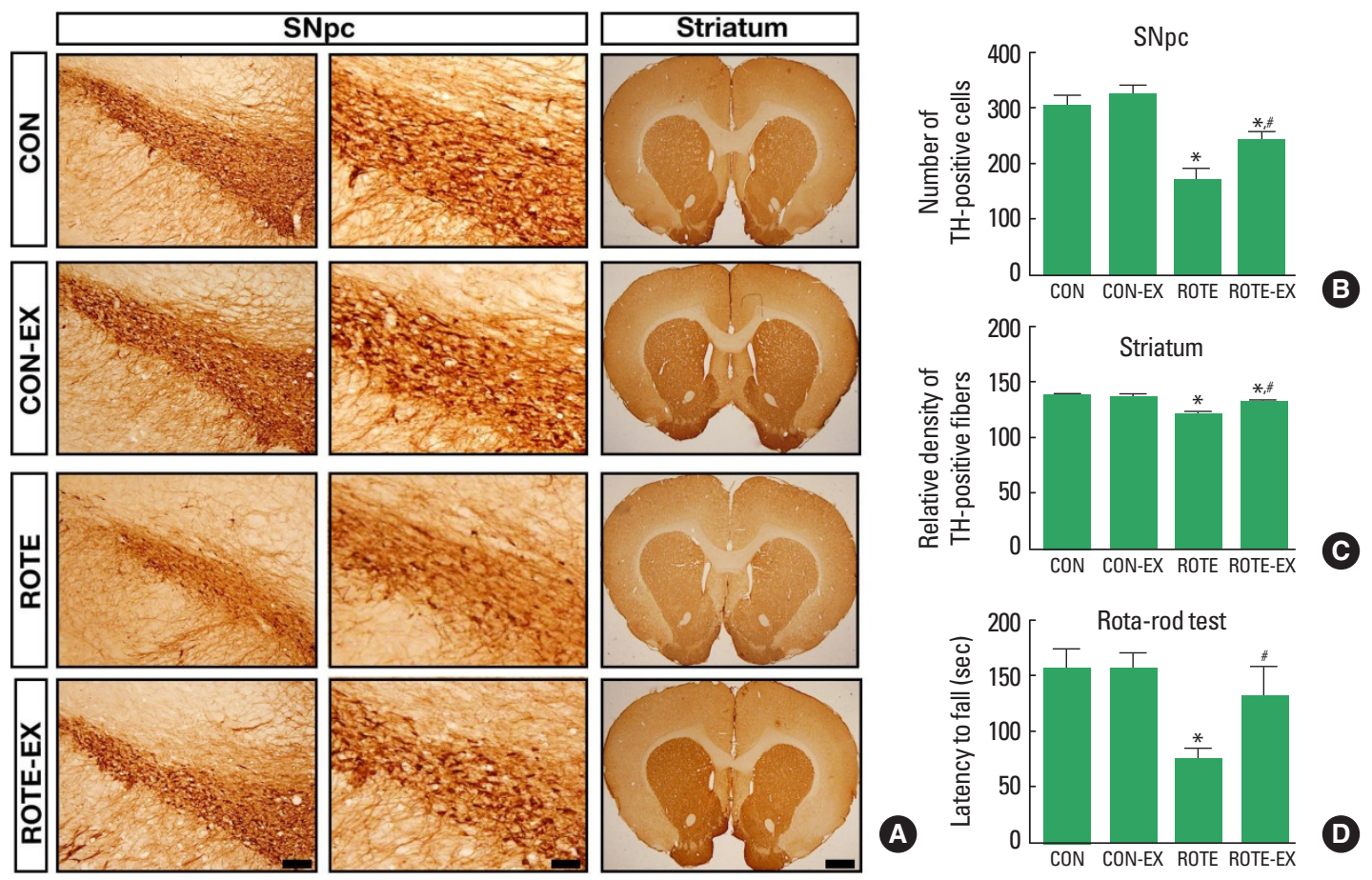

Fig. 1. Effects of treadmill exercise on tyrosine hydroxylase (TH) expressions in the substantia nigra pars compacta (SNpc; B) and the striatum (C), and on motor balance and coordination (rota-rod test; D). (A) Photomicrographs showing TH immunohistochemistry in the SNpc at low magnification (left panel, scale bar 200 um), at high magnification (middle panel, scale bar $50 \mu \mathrm{m}$ ), and in the striatum (right panel, scale bar $800 \mu \mathrm{m}$ ). CON, control group; CON-EX, control exercise group; ROTE, rotenone-injection group; ROTE-EX, rotenone-injection and exercise group. Data are presented as the means \pm standard error of the mean. ${ }^{*} P<0.05$ compared to the control group. ${ }^{\#} P<0.05$ compared to the rotenone-injected group.

$\mu \mathrm{m}$ square images of the striatum using an image analyzer (Multiscan, Fullerton, CA, USA).

\section{Immunohistochemistry for a-synuclein in the nigrostriatum}

The sections were rinsed in PBS and incubated in $1 \% \mathrm{H}_{2} \mathrm{O}_{2}$ for $30 \mathrm{~min}$ to block the endogenous peroxidase activity. After washing in PBS, the sections were incubated in blocking serum (10\% normal horse serum and $0.1 \%$ Triton X-100 in PBS) for $60 \mathrm{~min}$, followed by incubation in anti- $\alpha$-synuclein rabbit monoclonal antibody solution (1:500, Santa Cruz Biotechnology, Santa Cruz, CA, USA) for $24 \mathrm{hr}$ at room temperature. The sections were then incubated for $1 \mathrm{hr}$ in biotinylated anti-rabbit IgG secondary antibody (1:300; Vector Laboratories). The sections were subsequently incubated with avidin-biotin-peroxidase complex (Vector Laboratories) for $1 \mathrm{hr}$ at room temperature. Immunoreactivity was visualized by incubating the sections in a solution consisting of $0.05 \%$ 3,3-DAB and $0.02 \% \mathrm{H}_{2} \mathrm{O}_{2}$ in $50-\mathrm{mM}$ Tris buffer $(\mathrm{pH}, 7.6)$ for 3 $\min$. The sections were mounted on gelatine-coated slides and cover slipped with mounting medium.

\section{RESULTS}

\section{Latency in rota-rod test}

The latency to fall in the rota-rod test was decreased in the rotenone-induced Parkinson rats $(P<0.05)$. However, treadmill exercise increased the latency in the Parkinson rats $(P<0.05)$ (Fig. 1D).

\section{Expression of TH in nigrostriatum}

Expression of TH in substantia nigra pars compacta was decreased in the rotenone-induced Parkinson rats $(P<0.05)$. However, treadmill exercise increased TH expression in the Parkinson rats $(P<0.05)$ (Fig. 1B).

$\mathrm{TH}$ expression in striatum was decreased in the rotenone-induced Parkinson rats $(P<0.05)$. However, treadmill exercise increased TH expression in the Parkinson rats $(P<0.05)$ (Fig. 1C).

\section{Expression of a-synuclein in nigrostriatum}

Expression of $\alpha$-synuclein in substantia nigra was increased in the rotenone-induced Parkinson rats $(P<0.05)$. However, treadmill exercise decreased $\alpha$-synuclein expression in the Parkinson 


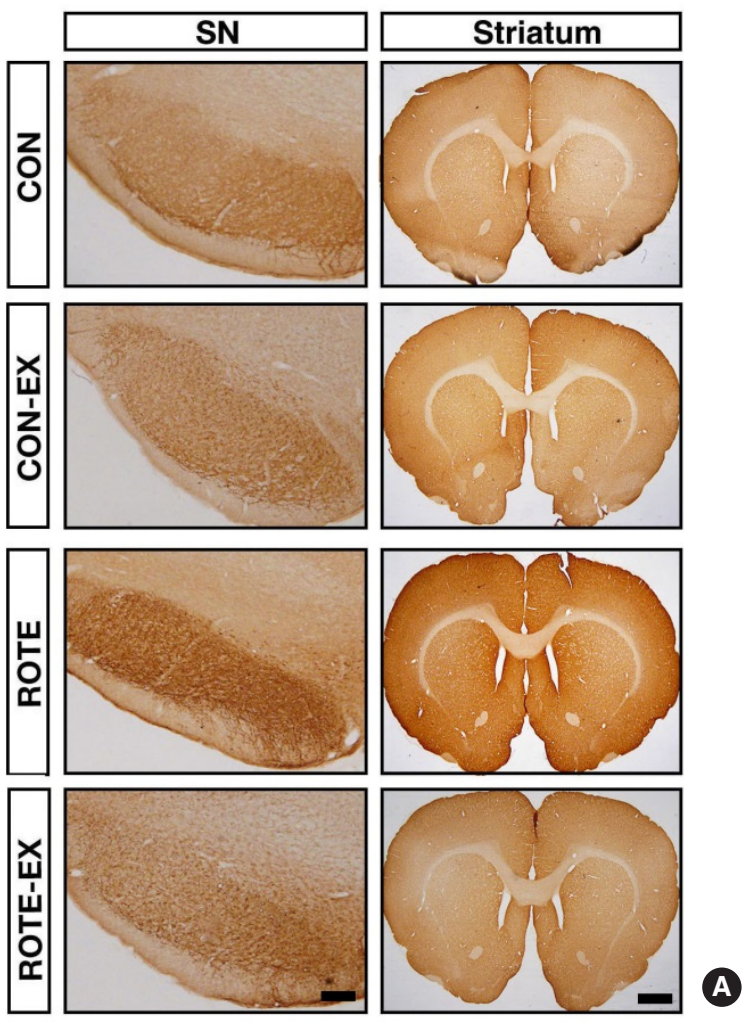

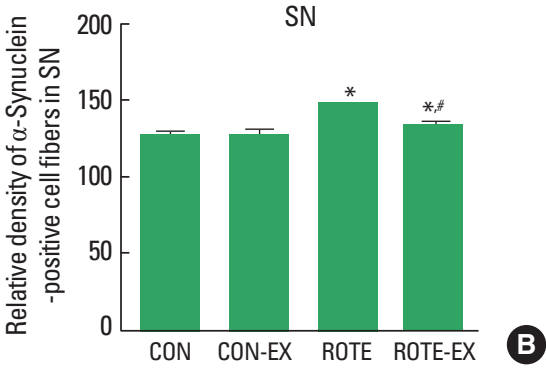

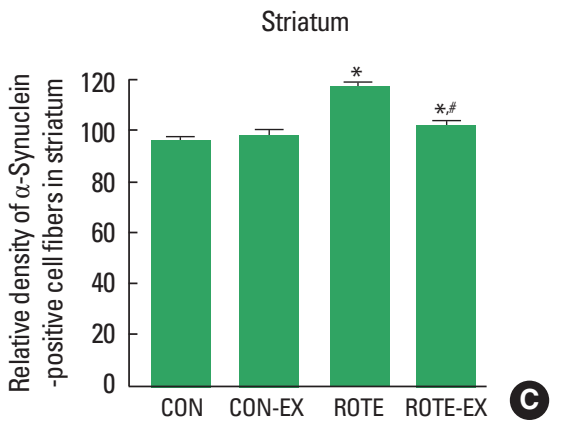

Fig. 2. Effects of treadmill exercise on the $\alpha$-synuclein expressions in the substantia nigra (SN; B) and striatum (C). (A) Photomicrographs showing $\alpha$-synuclein immunohistochemistry in the SN (scale bar $200 \mu \mathrm{m}$ ) and striatum (scale bar $800 \mu \mathrm{m}$ ). CON, control group; CON-EX, control exercise group; ROTE, rotenone-injection group; ROTE-EX, rotenone-injection and exercise group. Data are presented as the means \pm standard error of the mean. ${ }^{*} P<0.05$ compared to the control group. ${ }^{*} P<0.05$ compared to the rotenone-injected group.

rats $(P<0.05)$ (Fig. 2B).

Expression of $\alpha$-synuclein in striatum was increased in the rotenone-induced Parkinson rats $(P<0.05)$. However, treadmill exercise decreased $\alpha$-synuclein expression in the Parkinson rats $(P<0.05)$ (Fig. 2C).

\section{DISCUSSION}

Balance and strength training program improved equilibrium and strength in Parkinson disease patients (Toole et al., 2000). Forced limb-use prevented the behavioral and neurochemical deficits in 6-hydroxydopamine (6-OHDA)-induced Parkinson rats through increasing glial cell-line derived neurotrophic factor (GDNF) expression (Cohen et al., 2003). Disturbed motor coordination showed shortened latency in the rota-rod test and treadmill running lengthened this latency (Cho et al., 2016; Ji et al., 2015). In the present study, motor balance and coordination was disturbed by induction of rotenone-induced Parkinson disease, in contrast, treadmill exercise alleviated motor dysfunction in the ro- tenone-induced Parkinson rats. These results demonstrate that treadmill running improved motor function in Parkinson rats.

Oxidative stress is implicated in the aging brain and most of neurodegenerative diseases, such as Alzheimer disease, mild cognitive impairment, Parkinson disease, amyotrophic lateral sclerosis, and Huntington's disease (Mariani et al., 2005). TH expression in the cell bodies of the substantia nigra and TH expression in the fibers of the striatum were decreased in the 6-hydroxydapamine injected rats, in contrast, treadmill running enhanced the survival of dopaminergic neurons in the substantia nigra and their fibers in the striatum (Yoon et al., 2007). Cho et al. (2013) also showed that treadmill running alleviated nigrostriatal dopaminergic loss and improved short-term memory in 6-hydroxydapamine-induced Parkinson rats. In the present study, nigrostriatal dopaminergic loss of neurons and fibers was occurred by induction of rotenone-induced Parkinson disease, in contrast, treadmill exercise alleviated nigrostriatal dopaminergic loss of neurons and fibers in the rotenone-induced Parkinson rats. These results demonstrate that treadmill running preserved nigrostriatal dopa- 
minergic neurons and fibers in Parkinson rats.

a-Synuclein aggregates to form insoluble fibrils in pathological conditions characterized by Lewy bodies, such as Parkinson disease, dementia with Lewy bodies, and multiple system atrophy (Norris et al., 2004). These disorders are known as synucleinopathies. a-Synuclein is the primary structural component of Lewy body fibrils. The aggregation mechanism of $\alpha$-synuclein is uncertain, however, there is evidence of a structured intermediate rich in $\beta$-structure that can be the precursor of aggregation, and ultimately, Lewy bodies (Kim et al., 2007). In the present study, a-synuclein expression in the nigrostriatum was enhanced by induction of rotenone-induced Parkinson disease, in contrast, treadmill exercise suppressed $\alpha$-synuclein expression in the rotenone-induced Parkinson rats. These results demonstrate that treadmill running inhibited nigrostriatal formation of Lewy bodies in Parkinson rats.

In the present study, treadmill exercise improved motor function through preservation of nigrostriatal dopaminergic neurons and fibers and suppression of nigrostriatal formation of Lewy bodies in rotenone-induced Parkinson rats. Based on the present results, treadmill exercise may be used as the therapeutic strategy for delaying progression of Parkinson disease.

\section{CONFLICT OF INTEREST}

No potential conflict of interest relevant to this article was reported.

\section{ACKNOWLEDGMENTS}

This work was supported by the National Research Foundation of Korea Grant funded by the Korean Government (NRF-2013S1 A5A2A01018924).

\section{REFERENCES}

Berrios GE, Campbell C, Politynska BE. Autonomic failure, depression and anxiety in Parkinson's disease. Br J Psychiatry 1995;166:789-792.

Betarbet R, Sherer TB, MacKenzie G, Garcia-Osuna M, Panov AV, Greenamyre JT. Chronic systemic pesticide exposure reproduces features of Parkinson's disease. Nat Neurosci 2000;3:1301-1306.

Burkhardt CR, Weber HK. Parkinson's disease: a chronic, low-grade antioxidant deficiency? Med Hypotheses 1994;43:111-114.

Chen H, Zhang SM, Schwarzschild MA, Hernán MA, Ascherio A. Physical activity and the risk of Parkinson disease. Neurology 2005;64:664-
669.

Cho HS, Kim TW, Ji ES, Park HS, Shin MS, Baek SS. Treadmill exercise ameliorates motor dysfunction through inhibition of Purkinje cell loss in cerebellum of valproic acid-induced autistic rats. J Exerc Rehabil 2016;12:293-298.

Cho HS, Shin MS, Song W, Jun TW, Lim BV, Kim YP, Kim CJ. Treadmill exercise alleviates short-term memory impairment in 6-hydroxydopamine-induced Parkinson's rats. J Exerc Rehabil 2013;9:354-361.

Cohen AD, Tillerson JL, Smith AD, Schallert T, Zigmond MJ. Neuroprotective effects of prior limb use in 6-hydroxydopamine-treated rats: possible role of GDNF. J Neurochem 2003;85:299-305.

Ehringer $\mathrm{H}$, Hornykiewicz O. Distribution of noradrenaline and dopamine (3-hydroxytyramine) in the human brain and their behavior in diseases of the extrapyramidal system. Parkinsonism Relat Disord 1998;4:53-57.

Giasson BI, Jakes R, Goedert M, Duda JE, Leight S, Trojanowski JQ, Lee VM. A panel of epitope-specific antibodies detects protein domains distributed throughout human alpha-synuclein in Lewy bodies of Parkinson's disease. J Neurosci Res 2000;59:528-533.

Iwai A, Masliah E, Yoshimoto M, Ge N, Flanagan L, de Silva HA, Kittel A, Saitoh T. The precursor protein of non-A beta component of Alzheimer's disease amyloid is a presynaptic protein of the central nervous system. Neuron 1995;14:467-475.

Ji ES, Kim YM, Shin MS, Kim CJ, Lee KS, Kim K, Ha J, Chung YR. Treadmill exercise enhances spatial learning ability through suppressing hippocampal apoptosis in Huntington's disease rats. J Exerc Rehabil 2015;11:133-139.

Kim HY, Heise H, Fernandez CO, Baldus M, Zweckstetter M. Correlation of amyloid fibril beta-structure with the unfolded state of alpha-synuclein. Chembiochem 2007;8:1671-1674.

Mariani E, Polidori MC, Cherubini A, Mecocci P. Oxidative stress in brain aging, neurodegenerative and vascular diseases: an overview. J Chromatogr B Analyt Technol Biomed Life Sci 2005;827:65-75.

Miyai I, Fujimoto Y, Ueda Y, Yamamoto H, Nozaki S, Saito T, Kang J. Treadmill training with body weight support: its effect on Parkinson's disease. Arch Phys Med Rehabil 2000;81:849-852.

Norris EH, Giasson BI, Lee VM. Alpha-synuclein: normal function and role in neurodegenerative diseases. Curr Top Dev Biol 2004;60:17-54.

Schalow G, Pääsuke M, Jaigma P. Integrative re-organization mechanism for reducing tremor in Parkinson's disease patients. Electromyogr Clin Neurophysiol 2005;45:407-415.

Schuler F, Casida JE. Functional coupling of PSST and ND1 subunits in NADH:ubiquinone oxidoreductase established by photoaffinity labeling. Biochim Biophys Acta 2001;1506:79-87.

Talpade DJ, Greene JG, Higgins DS Jr, Greenamyre JT. In vivo labeling of 
mitochondrial complex I (NADH:ubiquinone oxidoreductase) in rat brain using [(3)H]dihydrorotenone. J Neurochem 2000;75:2611-2621.

Tillerson JL, Caudle WM, Reverón ME, Miller GW. Exercise induces behavioral recovery and attenuates neurochemical deficits in rodent models of Parkinson's disease. Neuroscience 2003;119:899-911.

Toole T, Hirsch MA, Forkink A, Lehman DA, Maitland CG. The effects of a balance and strength training program on equilibrium in Parkinsonism: a preliminary study. NeuroRehabilitation 2000;14:165-174. van Rooijen BD, van Leijenhorst-Groener KA, Claessens MM, Subramaniam V. Tryptophan fluorescence reveals structural features of alpha-synuclein oligomers. J Mol Biol 2009;394:826-833.

Yoon MC, Shin MS, Kim TS, Kim BK, Ko IG, Sung YH, Kim SE, Lee HH, Kim YP, Kim CJ. Treadmill exercise suppresses nigrostriatal dopaminergic neuronal loss in 6-hydroxydopamine-induced Parkinson's rats. Neurosci Lett 2007;423:12-17. 